\title{
Riscos do uso alternativo do cigarro eletrônico: uma revisão narrativa
}

\author{
Risks of alternative use of electronic cigarettes: a narrative review
}

\author{
Riesgos del uso alternativo de cigarrillos electrónicos: una revisión narrativa
}

Luana Soares Vargas ${ }^{1 *}$, Daniel Lopes Marques de Araújo ${ }^{1}$, Lorena Cota Noronha ${ }^{1}$, Lucas Antônio Avelar Carvalho', Matheus Fonseca Queiroz Mota ${ }^{1}$, Fernanda Pereira Alvarenga ${ }^{1}$, Glenda Mirelly de Oliveira Campos ${ }^{1}$, Ana Karoline Mendes Lima ${ }^{1}$, Vitória Gotelip Oliveira ${ }^{1}$, Ana Carolina Albernaz Barbosa1.

\section{RESUMO}

Objetivo: Avaliar os riscos do uso de cigarros eletrônicos como alternativa ao convencional. Revisão bibliográfica: Os cigarros eletrônicos têm sido utilizados como uma alternativa na cessação tabágica. Contudo, liberam quantidades consideráveis de nicotina e outras substâncias, muitas vezes não esclarecidas pelo fabricante, como o óleo de tetra-hidrocanabinol (THC). Além disso, a comercialização, importação e a publicidade no Brasil são proibidas, mas a venda é feita de forma clandestina. As evidências científicas sobre os danos causados pelos cigarros eletrônicos a longo prazo, são inconclusivas. Alguns estudos in vitro mostraram alterações na mucosa respiratória, assemelhando-se aos prejuízos causados pelos cigarros tradicionais. Em 2019, a Lesão Pulmonar Associada ao uso de Produtos com Cigarro Eletrônico ou Vaping (EVALI), foi descrita como uma doença respiratória aguda ou subaguda que pode evoluir ao óbito. Considerações finais: Portanto, ainda há dificuldade em classificar os cigarros eletrônicos como medicina auxiliar no uso de nicotina, porém é recomendado não usar seus produtos, principalmente com THC e obtidos de fontes informais.

Palavras-chave: Tabagismo, Cigarros eletrônicos, Nicotina.

\begin{abstract}
Objective: To assess the risks of using electronic cigarettes as an alternative to conventional. Bibliographic review: Electronic cigarettes have been used as an alternative in smoking cessation. However, they release considerable amounts of nicotine and other substances, often not clarified by the manufacturer, such as tetrahydrocannabinol oil (THC). In addition, commercialization, imports and advertising in Brazil are prohibited, but the sale is made clandestine. Scientific evidence on the long-term damage caused by e-cigarettes is inconclusive. Some in vitro studies have shown changes in the respiratory mucosa, similar to the damage caused by traditional cigarettes. In 2019, Lung Injury Associated with the Use of Products with Electronic Cigarette or Vaping (EVALI), was described as an acute or subacute respiratory disease that can progress to death. Final considerations: Therefore, it is still difficult to classify e-cigarettes as auxiliary medicine in the use of nicotine, however it is recommended not to use their products, mainly with THC and obtained from informal sources.
\end{abstract}

Key words: Smoking, Electronic cigarettes, Nicotine.

\section{RESUMEN}

Objetivo: Evaluar los riesgos del uso de cigarrillos electrónicos como alternativa a los convencionales. Revisión bibliográfica: Los cigarrillos electrónicos se han utilizado como alternativa para dejar de fumar. Sin embargo, liberan cantidades considerables de nicotina y otras sustancias, a menudo no aclaradas por el fabricante, como el aceite de tetrahidrocannabinol (THC). Además, la comercialización, importación y publicidad en Brasil están prohibidas, pero la venta se hace clandestinamente. La evidencia científica sobre el daño a largo plazo causado por los cigarrillos electrónicos no es concluyente. Algunos estudios in vitro mostraron cambios en la mucosa respiratoria, similares al daño causado por los cigarrillos tradicionales. En

\footnotetext{
${ }^{1}$ Centro Universitário Atenas (UniAtenas), Paracatu - MG. `E-mail: luanaasv@hotmail.com
} 
2019, la lesión pulmonar asociada al uso de productos con cigarrillos electrónicos o vapeo (EVALI) se describió como una enfermedad respiratoria aguda o subaguda que puede progresar hasta la muerte. Consideraciones finales: Por lo tanto, aún es difícil clasificar los cigarrillos electrónicos como medicina auxiliar en el uso de nicotina, sin embargo se recomienda no utilizar sus productos, especialmente con THC y obtenidos de fuentes informales.

Palabras clave: Tabaquismo, Cigarrillos electrónicos, Nicotina.

\section{INTRODUÇÃO}

Indícios apontam que o tabaco é uma planta originária dos andes bolivianos e era cultivada pelos indígenas para fins religiosos e medicinais. Os nativos a utilizavam de diversas formas: mascavam, bebiam, comiam, aspiravam e, principalmente, fumavam. Tal produto chegou ao Brasil através das migrações indígenas e os europeus tiveram contato com essa planta em 1492, com a chegada de Cristóvão Colombo à América. No século XVII, passou a ser um dos principais produtos de exportação português, sendo a sua venda impulsionada com a Proclamação da Independência do Brasil em 1822. Em 1903, surgiu nas terras brasileiras a primeira máquina de fabricação de cigarros já enrolados em papel trazida pelo português Albino Souza Cruz (HAJEK P, et al., 2019).

A utilização do tabaco e seus derivados acarreta a morte de milhões de pessoas a cada ano. De acordo com a Organização Mundial de Saúde, o tabagismo é a causa mais prevalente de óbitos evitáveis no mundo. Os dados estatísticos apontam que em 2030, se os números atuais mantiverem seu crescimento, o tabaco causará a morte de 8 milhões de indivíduos por ano e $80 \%$ será nos países subdesenvolvidos (RIGOTTI NA, 2018).

No Brasil, em 2015, estima-se que 40 bilhões de reais foram gastos com assistência médica por causa dessa prática. Isso representa $8,04 \%$ de todas as despesas em saúde. Os números de 2009 já mostravam que o dispêndio mundial relacionado ao tabaco seria de perdas de aproximadamente US $\$ 500$ bilhões ao ano, por consequências como diminuição da produtividade, doenças e mortes antecipadas (BARRETO IF, 2018).

As últimas décadas têm sido marcadas por uma luta incessante contra o tabagismo. Diversos programas e medidas governamentais são propagados com o intuito de diminuir ou até mesmo eliminar esse vício. Nesse contexto, os cigarros eletrônicos surgem como um empecilho, pois contribuem para a renormalização do hábito de fumar. No senso comum, eles são vistos como um mal menor e uma alternativa para vencer o tabagismo. Contudo, em diversos países, as propagandas por trás desses dispositivos são mais apelativas aos jovens e aos não fumantes, com o intuito de fidelizar novos clientes. Atrelado a isso está o fascínio pela inovação que estes produtos geram nos indivíduos, muitos vaporizadores possuem até mesmo funcionalidades atraentes, como controlar a seleção de músicas em outros aparelhos. Assim, esse cenário facilita a popularização de um produto que causa danos consideráveis à saúde (VILARDI BMR e VILARDI TMR, 2015).

Desse modo, o objetivo desta revisão foi avaliar os riscos do uso de cigarros eletrônicos como alternativa ao cigarro convencional.

\section{REVISÃO BIBLIOGRÁFICA}

\section{O que são os cigarros eletrônicos e como funcionam?}

Nos últimos anos, os cigarros eletrônicos têm sido utilizados como uma alternativa na cessação do tabagismo ou como um substituto do cigarro convencional. Contudo, segundo a OMS, não existe comprovação científica dos benefícios dessas práticas. Os cigarros eletrônicos são conhecidos como ecigarros/e-cigarettes ou Electronic Nicotine Delivery Systems (ENDS), e popularmente os usuários são chamados de vapers. São compostos por um bocal; um vaporizador ou inalador, em que o usuário suga o vapor; um cartucho ou atomizador, onde o líquido é armazenado e evaporado por meio do mecanismo de 
ativação da bateria de lítio recarregável; por um circuito microelétrico ou luz de indicação, ativado quando está em operação (MAIA LIH e NASCIMENTO EGC, 2015).

O uso do dispositivo é similar aos cigarros tradicionais. No ato da tragada, através de um botão ou pelo sensor de sucção, aciona um ciclo de aquecimento até atingir o ponto de ebulição do líquido, para que seja transformado em vapor. A temperatura pode atingir de $40-65^{\circ} \mathrm{C}$. Parte do vapor é liberada com a nicotina para o vape e a outra para o ambiente. Ao exalar ocorre o resfriamento, e o dispositivo só aquecerá novamente quando tragado. Um cartucho pode gerar de 10-250 jatos, o que pode corresponder, dependendo da marca, a 5-30 cigarros convencionais (RIGOTTI NA, 2018).

Tem sido notado que os fabricantes dos líquidos não informam a sua verdadeira composição, que geralmente contêm glicerina, propileno glicol, água, flavorizantes e nicotina, em que sua variação encontrada é de 16 até $22 \mathrm{mg} / \mathrm{ml}$, fator preocupante devido à toxicidade. Ainda, algumas avaliações químicas indicam nos cartuchos de nicotina a presença de substâncias potencialmente danosas, como formaldeído, acroleína, acetaldeído, metais pesados, compostos orgânicos voláteis e nitrosaminas derivadas do tabaco (CAVALCANTE TM, et al., 2017).

Além disso, os cigarros eletrônicos possuem muitos sabores e aromas diferentes, tornando-os mais atrativos, principalmente, para adolescentes. Os cartuchos e refis podem ser encontrados via internet ou em tabacarias. Também são fornecidos sabores dos atuais fabricantes de marcas famosas de cigarros convencionais. Alguns usuários os utilizam com outras substâncias, como cannabis, ervas, aromatizantes e vodca (BALS R, et al., 2019).

No mesmo viés, a Agência Nacional de Vigilância Sanitária (ANVISA) proibiu a comercialização, importação e a circulação de mensagens publicitárias de quaisquer dispositivos eletrônicos no Brasil. Essa resolução foi publicada em 2009 e seu objetivo era proteger os consumidores, pois ainda inexistiam estudos concretos que comprovavam que o uso desses dispositivos era seguro. Ademais, faz-se necessário a apresentação de estudos toxicológicos e testes científicos para que a ANVISA permita 0 uso de tais dispositivos caso haja alguma solicitação. Apesar dessa proibição, há conhecimento de que a venda de ENDS é feita de forma clandestina na internet e nas ruas (GRANA R, et al., 2014).

\section{Quais os malefícios que o cigarro tradicional traz aos fumantes?}

Muitas pessoas acham que o hábito de fumar é pelo simples fato da dependência a nicotina, porém estudos demonstram que envolve comportamentos, percepções, sensações, prazeres e sentimentos, relação entre estímulos ambientais e condicionamentos psicossociais. Além de prejuízos físicos, o vício pelo cigarro tradicional atinge valores socioculturais, uma vez que muitos indivíduos começam a fumar antes de vinte anos, e valores socioeconômicos, já que interfere nas despesas pessoais. Ademais, existem várias doenças relacionadas, atingindo principalmente os aparelhos respiratório, como doença pulmonar obstrutiva crônica e agravamento da asma; o cardiovascular acarretando aterosclerose e acidente vascular cerebral, o digestivo desenvolvendo refluxo gastroesofágico, úlcera péptica e cirrose hepática, e o genitourinário ocasionando disfunção erétil e infertilidade (GLANTZ SA e BAREHAM DW, 2018).

A fumaça do cigarro possui milhares de compostos químicos, sendo alguns envolvidos com desenvolvimento de neoplasias. A fase gasosa da fumaça contém monóxido de carbono, formaldeído, amônia, acetaldeído e a fase particulada contém a nicotina. A combustão do produto forma várias substâncias cancerígenas como arsênio e benzopireno, além de resíduos de agrotóxicos, radioativos e até raticidas. (ARRUDA ITS e MENDONÇA TGL, 2019).

Além disso, o tabagismo provoca neoplasias malignas na cavidade oral, faringe, esôfago, estômago e pulmões. Na gravidez e no feto pode provocar abortamento espontâneo, descolamento prematuro da placenta, menor peso ao nascer e malformações congênitas. Ainda influencia em outras doenças, como envelhecimento da pele, pigmentação melânica, doença periodontal, halitose, estomatites, queda das defesas imunitárias. Ademais, o uso do cigarro convencional, conforme a Décima Revisão de Classificação Internacional de Doenças (CID10), é um problema classificado como transtorno mental e pode influenciar no comportamento do indivíduo devido a utilização da substância psicoativa (FERREIRA JMR, et al., 2015). 


\section{Os cigarros eletrônicos acarretam prejuízos à saúde?}

As evidências científicas sobre os danos causados pelos cigarros eletrônicos, a longo prazo, são inconclusivas. Tal cenário acontece devido à recente criação e popularização desses produtos. $O$ fato de os estudos epidemiológicos longitudinais serem insuficientes também contribui para essa incógnita. Alguns estudos in vitro mostraram aumento do stress oxidativo, apoptose e alteração na função dos cílios da mucosa respiratória. Esses prejuízos são semelhantes aos causados pelos cigarros convencionais, porém de forma mais amena. Uma outra pesquisa em humanos, 30 fumantes versus controle, mostrou um aumento da impedância respiratória e da resistência das vias aéreas devido à inalação aguda de vapor dos cigarros eletrônicos (KNORST MM, et al., 2014) .

Por outro lado, foi examinado 41 fluidos de recarga de cigarros eletrônicos para avaliar a citotoxicidade. Como parâmetro foram utilizados 3 tipos de células: fibroblastos pulmonares humanos, células-tronco embrionárias humanas e células-tronco neurais de camundongos. Constatou-se que as células-tronco são mais sensíveis aos efeitos dos vapores e que, devido a isso, as preocupações com as grávidas fumantes de e-cigarros aumentaram, por causa dos efeitos deletérios sobre o feto. Os autores concluíram que a citotoxicidade não estava relacionada a nicotina, mas ao número e a concentração dos aromas utilizados (KUSTERS D, et al., 2015).

Relatos de usuários indicaram irritação na boca e na garganta, tosse, dores de cabeça, dispneia e vertigem. Estudos mostram que a exposição ao vapor dos e-cigarros pode acarretar danos ao ácido desoxirribonucléico (DNA) através da quebra das fitas duplas. Os resultados afirmam que eles ainda são causados na ausência de nicotina, o que mostra o poder lesivo dos extratos aromatizantes. A quebra da fita dupla pode trazer alterações irreparáveis e aberrações genômicas. Outrossim, as linhas celulares expostas ao vapor apresentaram parada em G1 e G2, demonstrando alteração do perfil do ciclo celular (SILVA ALO e MOREIRA JC, 2019).

Um estudo revela que os dispositivos eletrônicos podem acarretar a aceleração dos batimentos cardíacos e outros efeitos subjetivos. Ademais, um ponto importante é o aumento da incidência de intoxicação entre as crianças pela ingestão acidental dos seus cartuchos. Somado a isso, há o risco de explosão desses e-cigarros enquanto estão carregando, fato que já causou ferimentos e incêndios. Portanto, esses fatores configuram como riscos adicionais à saúde e ao bem-estar das pessoas (POLOSA R, et al., 2014).

\section{Poderá ser admitido o seu uso na cessação tabágica?}

O abandono da prática tabágica tem sido algo almejado por muitos indivíduos. A renúncia dos cigarros convencionais acarreta diversos benefícios à saúde, visto que esse hábito é imensamente deletério ao organismo. Apesar dessa vontade por parte dos fumantes, há uma grande dificuldade em abandonar tal vício a longo prazo. Estudos revelam que aqueles que tentam se livrar dessa prática sem um suporte profissional, $50 \%$ das vezes não conseguem parar nem por uma semana e menos de $5 \%$ ficam abstinentes durante um ano após o abandono do tabagismo (ST.HELEN G, et al., 2017).

A maior parte da dependência está associada a nicotina, contudo, aspectos sensoriais e comportamentais influenciam na permanência do tabagismo. Atitudes como o ato de segurar o cigarro, as tragadas, o "arranhão" na parte de trás da garganta funcionam como estimulantes ao vício. Por conseguinte, as terapêuticas de substituição nicotínica também não configuram uma alternativa poderosa contra o vício, devido ao fato de não proporcionarem outras situações que estão relacionadas à dependência. Assim, os e-cigarretes surgem como uma solução plausível (BOLÉO-TOMÉ JP, et al., 2019).

Os cigarros eletrônicos são vistos como uma forma de abandono ao tabagismo tradicional, pois a sua similaridade com os cigarros convencionais amenizaria os efeitos que o simples ato de fumar causa, como as sensações que acompanham o fumo, porém, supostamente sem os efeitos adversos à saúde. Um estudo randomizado feito com 886 participantes comparou o uso do cigarro eletrônico com a terapia de substituição de nicotina no abandono do tabagismo e demonstrou que foram mais eficazes. Contudo, ainda carecem de estudos que comprovem, de forma assertiva, a sua eficácia na cessação tabágica (KALININSKIY A, et al., 2019). 
Porém, alguns estudos atuais mostraram que o produto já possui efeitos adversos notificados, como irritação da mucosa bucal e ocular, obstrução leve das vias aéreas superiores, além de reações por abandono recente, como vertigem, náuseas e cefaleia; análogas aos sintomas que o cigarro tradicional provoca. Além disso, um cartucho libera quantidades consideráveis de nicotina, e ainda expõem os usuários a altos níveis de partículas ultrafinas e outras substâncias, muitas vezes não esclarecidas pelo fabricante, que podem aumentar consideravelmente os riscos de patologias pulmonares, cardiovasculares e não-cancerígenas, podendo os ENDS serem associados a mais da metade de mortes provocadas pelo fumo, sendo similares aos danos dos cigarros convencionais (RIGOTTI NA, 2018).

\section{O que é EVALI?}

A Lesão Pulmonar Associada ao Uso de Produtos com Cigarro Eletrônico ou Vaping (EVALI) ou Lesão Pulmonar Associada a Vaping (VAPI), descrita em 2019, é uma doença respiratória aguda ou subaguda que pode ser grave e com risco de vida. Sua patogênese ainda não é conhecida, mas há achados patológicos de pneumonite fibrinosa aguda, dano alveolar difuso ou pneumonia em organização, geralmente bronquiolocêntrica e acompanhada de bronquiolite (WEEN MP, et al., 2021).

Além disso, há pneumonia eosinofílica aguda, hemorragia alveolar difusa, pneumonia lipóide e doença pulmonar intersticial por bronquiolite respiratória, sugerindo que mais de um mecanismo de lesão pode estar envolvido. O principal fator de risco é o uso de cigarro eletrônico, mas ainda nenhum componente isolado foi identificado que seja comum a todos os casos. Dessa forma, a apresentação de uma doença respiratória sem quaisquer outros fatores conhecidos, exceto a história vaping, deixa um amplo diagnóstico diferencial (RAJA J, et al., 2021).

Na University of Rochester Medical Center (Rochester, NY, USA), relataram 12 casos tratados por suspeita de EVALI entre 6 de junho de 2019 e 15 de setembro de 2019. Dez (83\%) pacientes apresentaram febre, dispneia, vômitos e nove (75\%) tosse. 11 (92\%) pacientes fizeram uso de cartuchos de cigarro eletrêonico contendo óleo de tetra-hidrocanabinol (THC), o principal componente psicoativo da cannabis (HAJEK P, et al., 2019).

Os Estados Unidos estão enfrentando um surto sem precedentes da doença. O Departamento de Saúde Pública de Illinois (IDPH) realizou uma pesquisa pública online entre setembro e outubro de 2019 com usuários de cigarro eletrônico no estado. Entre 4.631 participantes da pesquisa, $94 \%$ relataram usar qualquer cigarro eletrônico com nicotina nos últimos 3 meses; $21 \%$ com produtos contendo THC; e $11 \%$ usaram ambos. A prevalência do uso de produtos contendo THC foi maior entre os entrevistados com idades entre 18 e 24 anos (36\%). Dentre os participantes, 66 eram pacientes EVALI, entre 18 e 44 anos, entrevistados como parte da investigação em andamento do surto, e foram comparados ao subconjunto de 519 participantes, com o mesmo intervalo de idade, que relataram usar THC. Os pacientes EVALI apresentaram maiores chances de uso exclusivo e frequente (mais de cinco vezes por dia) de produtos com THC e com obtenção de fontes informais, como um revendedor ou de um amigo (FRIEDMANAS e TAM J, 2021).

Além disso, o Departamento de Saúde e Serviços Humanos dos EUA (CDC) em parceria com Food and Drug Administration (FDA) pesquisaram em 10 estados americanos no mês de outubro de 2019 e observaram que $86 \%$ dos 867 pacientes EVALI relataram usar produtos com THC nos 3 meses anteriores ao início dos sintomas. As análises de amostras de produtos pelo FDA e pelos laboratórios de saúde pública identificaram constituintes potencialmente patológicos, como acetato de vitamina E, óleo de triglicerídeos de cadeia média (óleo MCT) e outros lipídios. O acetato de vitamina E, pode ser usado como aditivo nos produtos de vaping e como agente espessante em produtos THC4. Portanto, essas descobertas reforçam as recomendações atuais de não usar produtos de cigarro eletrônico, principalmente que contenham THC e sejam obtidos de fontes informais (WEEN MP, et al., 2021).

\section{CONSIDERAÇÕES FINAIS}

Portanto, os cigarros eletrônicos configuram um entrave na cessação tabágica, pois estimulam a renormalização do hábito de fumar e causam danos à saúde. As evidências sobre tais danos a longo prazo 
ainda são inconclusivas. Porém, os prejuízos já notificados assemelham-se aqueles acarretados pelos cigarros tradicionais. Outrossim, estudos recentes mostram a sua associação com a EVALI. Entretanto, a inexistência de normas técnicas e fiscalização sobre a produção e venda, tornam sua utilização mais acessível, mesmo ainda sendo proibida pela ANVISA. Assim, este trabalho visou elucidar as consequências do uso do cigarro eletrônico como uma alternativa para o cigarro tradicional.

\section{REFERÊNCIAS}

1. ARRUDA ITS, MENDONÇA TGL. Câncer de pulmão: efeitos da inalação passiva dos compostos químicos do cigarro. Revista Saúde e Ciência online, 2019; 8(2): 66-72.

2. BALS R, et al. Electronic cigarettes: A task force report from the European Respiratory Society. European Respiratory Journal, 2019; 53(2): 1-24.

3. BARRETO IF. Tabagismo, cigarros eletrônicos e redução de danos: uma revisão narrativa. Revista Ciências Em Saúde, 2018; 8(1): 18.

4. BOLÉO-TOMÉ JP, et al.The doctor, the smoking patient and the challenge of electronic cigarettes. Acta Medica Portuguesa, 2019; 32(7-8): 477-82.

5. CAVALCANTE TM, et al. Conhecimento e uso de cigarros eletrônicos e percepção de risco no Brasil: Resultados de um país com requisitos regulatórios rígidos. Cadernos de Saude Publica, 2017; 33(3): 1-11.

6. FERREIRA JMR, et al. Cigarro Eletrónico : Posição da Sociedade Portuguesa de Pneumologia. Revista Científica da Ordem dos Médicos, 2015; 28(5): 548-51.

7. FRIEDMANAS, TAM J. E-Cigarettes: Matching Risks With Regulations. American Journal of Preventive Medicine, 2021; 60(1): 146-150.

8. GLANTZ SA, BAREHAM DW. E-Cigarettes: Use, Effects on Smoking, Risks, and Policy Implications. Annual Review of Public Health, 2018; 39(1): 215-35.

9. GRANA R, et al. E-cigarettes: A scientific review. Circulation, 2014; 129(19): 1972-86.

10. HAJEK P, et al. A randomized trial of e-cigarettes versus nicotine-replacement therapy. New England Journal of Medicine, 2019; 380(7): 629-37.

11. KALININSKIY A, et al.E-cigarette, or vaping, product use associated lung injury (EVALI): case series and diagnostic approach. The Lancet Respiratory Medicine, 2019; 7(12): 1017-26.

12. KNORST MM, et al.Cigarro eletrônico: O novo cigarro do século 21? Jornal Brasileiro de Pneumologia, 2014; 40(5): 564-73.

13. KUSTERS D, et al. Percepção De Riscos E Benefícios Do Cigarro Eletrônico Versus O Tradicional. Revista Fatec Sebrae em debate - gestão, tecnologias e negócios, 2015; 2(03): 03.

14. MAIA LIH, NASCIMENTO EGC. Percepções E Dificuldades De Fumantes Na Cessação Do Hábito De Fumar. Revista da Universidade Vale do Rio Verde, 2015; 13(2): 105-16.

15. POLOSA R, et al. Effectiveness and tolerability of electronic cigarette in real-life: A 24-month prospective observational study. Internal and Emergency Medicine, 2014; 9(5): 537-46.

16. RAJA J, et al. Smoke and Heart Should Stay Apart: A Look at E Cigarettes and Other Alternatives to Conventional Cigarettes, and Their Impact on Cardiovascular Health. Current Problems in Cardiology, 2021; 46(3): 100640.

17. RIGOTTI NA. Balancing the benefits and harms of e-cigarettes: a National Academies of Science, Engineering, and Medicine report. Annals of internal medicine, 2018; 168(9): 666-7.

18. SILVA ALO, MOREIRA JC. A proibição dos cigarros eletrônicos no Brasil: sucesso ou fracasso? Ciência \& Saúde Coletiva, 2019; 24(8): 3013-24.

19. ST.HELEN G, et al. Impact of e-liquid flavors on nicotine intake and pharmacology of e-cigarettes. Drug and Alcohol Dependence, 2017; 178(May): 391-98.

20. VILARDI BMR, VILARDI TMR. Cigarros eletrônicos: sim ou não? Faculdade de Odontologia de Lins/Unimep, 2015; 25(2): 76-7.

21. WEEN MP, et al. E-cigarettes and health risks: more to the flavor than just the name. American Physiological Society, 2021; 320(4): L600-L614. 